\title{
Different Nanotechnology Approaches for Ciprofloxacin Delivery Against Multidrug-Resistant Microbes
}

\author{
Ashagrachew Tewabe Yayehrad $\mathbb{D}^{1,2}$, Gebremariam Birhanu Wondie $\mathbb{D}^{\prime}$, Tesfa Marew' \\ 'Department of Pharmaceutics and Social Pharmacy, School of Pharmacy, College of Health Sciences, Addis Ababa University, Addis Ababa, Ethiopia; \\ ${ }^{2}$ Department of Pharmacy, College of Medicine and Health Sciences, Bahir Dar University, Bahir Dar, Ethiopia \\ Correspondence: Ashagrachew Tewabe Yayehrad, Tel +251912960525, Email ashutewabe@gmail.com
}

\begin{abstract}
The percentages of organisms exhibiting antimicrobial resistance, especially resistance to multiple antibiotics, are incessantly increasing. Studies investigated that many bacteria are being resistant to ciprofloxacin. This review addresses the current knowledge on nano-based ciprofloxacin delivery approaches to improve its effectiveness and overcome the resistance issues. Ciprofloxacin delivery can be modified by encapsulating with or incorporating in different polymeric nanoparticles such as chitosan, PLGA, albumin, arginine, and other organic and inorganic nanostructure systems. Most of these nano-approaches are promising as an alternative strategy to improve the therapeutic effectiveness of ciprofloxacin in the future.
\end{abstract}

Keywords: antimicrobial resistance, ciprofloxacin, ciprofloxacin resistance, nanotechnology

\section{Introduction}

Antibiotics are considered the most important advances in the history of modern medicine due to their phenomenal impact on reducing human morbidity, mortality, and economic losses. However, bacteria that challenge not only single but multiple antibiotics have become increasingly common through time. Antimicrobial resistance (AMR) is now the most important challenge in the fight against infectious diseases which is associated with inappropriate use and abuse of the drugs. ${ }^{1}$ The number of microbial strains resisting, the geographical coverage, and the extent of resistance toward multiple antibiotics are accelerating. Many drugs with known susceptibility to antibiotics therapy are now returning in new habits as resistant to those therapies. ${ }^{2}$

Quinolones are among great achievements in the antibiotic discovery. The first groups possessing a narrow range of action and unfavorable drug kinetics rose in the early 1960s. Through time, new quinolones have been developed with improved efficacy and spectrum. Nowadays, they are being widely prescribed for a variety of infectious diseases even though AMR is becoming a tackle against their effectiveness. ${ }^{3}$ They are known to act by inhibiting type 2 bacterial DNA topoisomerases, DNA gyrase, and topoisomerase IV which are the commonest areas of quinolone resistance now. They are classified with generations as first generation (nalidixic acid), second generation (norfloxacin, ciprofloxacin, ofloxacin, enoxacin, lomefloxacin), third generation (moxifloxacin, levofloxacin, gatifloxacin, sparfloxacin), and fourth generation (trovafloxacin). The first generation was effective against Gram-negative bacteria excluding Pseudomonas species but the spectrum broadens toward the new generations. ${ }^{4}$ Ciprofloxacin is used for the treatment of a wide range of infections, such as complicated urinary tract infections, typhoid, bone or joint infections, shigellosis, cystic fibrosis, chronic suppurative otitis media, prostatitis, and epididymo-orchitis. It is one of the topmost selling antibiotics being available at a cheaper cost and with different drug delivery systems like: oral tablets, eye drops, suspensions, ointments, infusion, in-situ gel, hydrogels, films, microbeads, elastic liposomes, floating matrix tablets, ocuserts, and niosomal cream. ${ }^{5}$

Even though a substantial and momentary reduction of infections caused by resistant bacteria was attained by quinolones, concerns about treatment failure and bacterial resistance appeared shortly after their innovation. ${ }^{6,7}$ Alarms regarding the suitable use of fluoroquinolones and the quality of their pharmaceutically equivalent preparations have been knocking after the therapeutic 
failures and amplified bacterial resistance were reported. ${ }^{4}$ Onwards, measures become in need to combat this resistance risk and get the most out of their effectiveness. Research and feedback mechanisms, antimicrobial stewardship programs (ASP), susceptibility testing, and targeted clinical response are among the approaches toward fighting AMR. The development of new antibiotics and discovery of novel drug delivery systems should also be considered. To be successful, determined efforts of all members of the society are necessary. ${ }^{8}$ With the growth of innovation and technology, there has been much interest in possible alternative antimicrobial therapies through nanotechnology. The scenarios for conjugates or combinations of nanomaterials (NMs) and the conventional antibiotic drugs for targeting and combating microbial resistance are in a careful optimism. ${ }^{9}$ Nanomaterials can carry or transport antimicrobials; deliver them into the target, or ultimately act synergistically by their inherent antimicrobial activity. Moreover, nanoparticles (NPs) may contest the mechanisms of bacterial drug resistance with different mechanisms. They can inhibit some important bacterial processes like biofilm formation. ${ }^{10}$ In this review, the different NMs and associated nanotechnology-based ciprofloxacin delivery approaches against resistant microbes have been discussed.

\section{Resistance Challenges of the Conventional Ciprofloxacin Delivery Ciprofloxacin-Resistant Problematic Groups and Challenges}

Ciprofloxacin has been shown to be active against various Gram-positive and Gram-negative bacterial isolates such as: E. coli, H. influenzae, other H. spp., N. gonorrhoeae, N. meningitides, Moraxella catarrhalis, K. pneumoniae, Legionella pneumophila, Moraxella catarrhalis, Proteus mirabilis, and P. aeruginosa, methicillin-susceptible S. aureus (MSSA), S. pneumoniae, S. epidermidis, E. faecalis, and S. pyogenes both in vitro and in vivo. ${ }^{4}$ It has been used successfully since 1987 and included in the WHO's essential medicines list. ${ }^{11}$ However, an increasing proportion of bacterial clinical isolates are being resistant to ciprofloxacin ${ }^{6}$ that induces higher rates of hospitalization, greater length of hospital stay, and higher rates of illness and death. ${ }^{12}$

Ciprofloxacin replaced aminoglycosides (due to their resistance) as the drug of choice against keratitis caused by P. aeruginosa. However, due to its overuse, there has been a considerable increase in ciprofloxacin resistance among P. aeruginosa isolates (from $<1 \%$ in 1994 , to $4 \%$ in 1998 , finally to $29 \%$ in 2003 , and exhibit multidrug-resistant (MDR) character now). Fluoroquinolones were recommended as a drug of choice to treat gonococcal infections in 1993. In 1997, the first resistant strains were reported in Hong Kong and Philippines. In 2004, fluoroquinolone was no longer recommended for treatment due to the increased resistance and declined sensitivity of the causative agent. As a result, cephalosporins were replaced in the place of fluoroquinolones for treating gonococcal infections. Table 1 summarizes the review by Ahmad et al on ciprofloxacin-resistant problematic groups of bacteria. ${ }^{13}$

Table I Ciprofloxacin-Resistant Problematic Bacterial Groups

\begin{tabular}{|c|c|c|c|c|}
\hline S. No & Study Area & Study Period & Group of Bacteria & Results \\
\hline I. & Brazil & $2009-2013$ & Salmonella & $43 \%$ \\
\hline 2. & Japan & 1992 & N. gonorrhoeae & $40 \%$ \\
\hline 3. & India & $1990-2000$ & $\gg$ & Most \\
\hline 4. & South Africa & 2004-2007 & $\gg$ & Increased: $7 \%$ to $32 \%$ \\
\hline 5. & Kenya & $2007-2009$ & $\gg$ & Increased: $9.5 \%$ to $50 \%$ \\
\hline 6. & Europe & 2013 & $\gg$ & $53 \%$ \\
\hline 7. & Latin America & $2004-2010$ & $\gg$ & $5-40 \%$ \\
\hline 8. & Pittsburgh & $|996-200|$ & S. aureus & Increased: $8 \%$ to $20.7 \%$ (MSSA), $55.8 \%$ to $83.7 \%$ (MRSA) \\
\hline 9. & Asian countries & $1980 s-2010 s$ & Campylobacters & 77-99\% \\
\hline 10. & South Africa & & $\gg$ & $91 \%$ \\
\hline 11. & United Arab Emirates & & $\gg$ & $85.4 \%$ \\
\hline 12. & Spain & $1993-2003$ & $\gg$ & $52.2 \%$ \\
\hline
\end{tabular}




\section{Mechanism for Development of Resistance}

Ciprofloxacin resistance in the majority of bacterial isolates is from chromosomal mutations and/or reduced drug accumulation due to overexpression of efflux pump systems. ${ }^{14}$ The efflux pump system reduces the intracellular concentrations to sub-lethal levels, while point mutations reduce drug binding to specific enzymatic sites, decreasing activity. In this regard, fluoroquinolones are prevented from binding to the enzymes involved in DNA replication, including DNA gyrase and DNA topoisomerase IV. ${ }^{12}$ Genetic mutations take the vast proportion of bacterial resistances. GyrAB and parCE are target genes of $P$. aeruginosa for ciprofloxacin action and their mutation reduced the affinity of DNA gyrase or topoisomerase for the drug. ${ }^{11}$ In Egypt, double mutations $(1$ gyrA/1 parC $)$ and three mutations $(2$ gyrA/1 parC) were found in different bacterial species like $E$. coli and $K$. pneumoniae. Plasmid-mediated quinolone resistance is also the other genetic related resistance for ciprofloxacin. Under this group, genes like aac( $\left.6^{\prime}\right)-I b-c r$ of $E$. coli, qnrB/aac $\left(6^{\prime}\right)-I b-c r$ and $q n r S$ of $K$. pneumoniae, qnrB of $P$. aeruginosa, and qnrS of $A$. baumannii have been reported. ${ }^{14}$ Ciprofloxacin resistance toward $S$. aureus is due to: 1) point mutations on encoding the subunits of topoisomerase IV and DNA gyrase; and 2) efflux by the membrane-associated protein which actively transports fluoroquinolones out of the bacterial cell. ${ }^{15}$ Mutation appears to be the only mechanism to acquire resistance in $M$. tuberculosis and some P. aeruginosa infections. P. aeruginosa contains 12 efflux pumps and four of these are known to efflux fluoroquinolones. E. coli resistance to ciprofloxacin, both in vivo and in vitro, requires mutation. ${ }^{16,17}$ Fluoroquinolone resistance to Salmonella isolates includes mutations, active efflux, and plasmid-mediated protection of target topoisomerases. The acrB, soxS, marA, ramA, and rob were characterized as ciprofloxacin-resistant Salmonella enterica serotype Enteritidis mutant genes. ${ }^{18}$ Whereas, resistance to $N$. gonorrhoeae, Campylobacter spp., and Salmonella can be chromosomally as well as plasmid-mediated and bacterial biofilm formation. ${ }^{13}$

\section{Factors Associated with Ciprofloxacin Resistance}

Indiscriminate use in routine clinical practices, unnecessary consumption during viral infections, and drug overuse are the determinant factors for ciprofloxacin and other antibiotics resistance. Clinically, three major factors are associated with increasing resistance to fluoroquinolones: 1) under-dosing, ie, use of a slightly potent drug whose MIC is scarcely reached in serum or infected tissues; 2) overuse or misuse of drugs that encourage resistant mutants; and 3) failure to timely identify and reply to changes in microbial susceptibilities. ${ }^{12}$ Prior use history and higher age, high intake of pork and chicken, as well as the concomitant prescription of calcium supplements or proton pump inhibitors with fluoroquinolones were reported as contributing factors for resistance to ciprofloxacin in UTIs. ${ }^{7}$ The accessibility and consumption of inferior-standard and counterfeit ciprofloxacin formulations, especially in the developing countries, is another factor which has contributed to the increased risk of therapeutic failure and drug resistance. The overuse or misuse of ciprofloxacin is likely to be due to widespread availability of generic versions of ciprofloxacin. ${ }^{4}$

\section{Strategies to Minimize or Counter Ciprofloxacin Resistance}

Historically, several approaches have been employed to address AMR. These include: 1) the use of a newer more potent antimicrobials; 2) continuing to use older agents, preserving the activity of the new drugs; 3) antibiotic cycling or rotation, with a predefined period, usually 3 months; 4) combination therapy; and 5) pharmacokinetic and pharmacodynamic data-based prescription have been exploited for years. Evidence-based antimicrobial selection must be implemented by clinicians. ${ }^{12}$ Loading ciprofloxacin with novel approaches to target the site of infection and use of combination therapies with other anti-Pseudomonas agents are also recommended as possible complementary strategies. ${ }^{11}$ Reserpine, a well-known mammalian MDR pump inhibitor, has resulted in suppression of resistance in S. aureus and S. pneumoniae strains when used in combination with ciprofloxacin. Boeravinone B 66, an inhibitor of the S. aureus multidrug efflux pump NorA, was recently shown to act synergistically with ciprofloxacin to inhibit biofilm formation by $S$. aureus. ${ }^{13}$ New drug development alone is now becoming insufficient for safeguarding the advancement in medicine due to the failure of addressing limitations associated with poor pharmacokinetics, incomplete targeting, and toxicity. Nowadays, the application of nanostructures that have intrinsic antimicrobial potential or nanocarriers that can modify the transport and delivery of the antibiotic have been presented as new strategies and defense mechanisms against MDR infectious organisms. ${ }^{9,19}$ Application of nanotechnology in the pharmaceutical field offers new advancements for developing novel 
formulations from the diverse types of nanoparticles (NPs) considering their different sizes and shapes with their versatile antimicrobial properties. These NPs can bring a hopeful solution as they can directly target the bacteria by themselves or can act as drug carrier systems. ${ }^{10}$

\section{Nanomaterials: Alternative Delivery Systems for AMR Nanomaterial Preparation and Mechanisms in Countering AMR}

Nanotechnology is an innovative science that utilizes NMs with dimensions from 1-1000 nm. Several NMs are already used as broad-scale antimicrobial agents and carriers of antimicrobial agents in consumer products. ${ }^{9}$ However, the practical applicability of different nanocarriers has been limited due to their insufficient biocompatibility, lower sensitivity (with respect to temperature and $\mathrm{pH}$ ), and lack of complete biodegradability. ${ }^{20}$ Antibiotic delivery with NPs results in improved bioavailability by enhancing aqueous solubility, increasing resistance time in the body, and targeting specific site of action. ${ }^{21}$ Although the nanotechnology-associated high costs make the conventional therapy preferable, these platforms might be of utmost importance with their impact on cell wall permeability, efflux activity, reactive species formation, and metabolism and reproduction inhibition. ${ }^{10}$

NMs can be incidental (byproduct of industrial and natural processes), engineered, or natural which may be produced from living organisms and plants. ${ }^{22}$ They are prepared and characterized for their properties in different ways. Metallic NPs are mainly synthesized by either reducing the particle size by mechanical or chemical methods or by green synthesis using bacteria, plant extract, and fungus. Polymeric NPs are prepared by cross linking, polymerization methods, and polymer precipitation methods. Table 2 states the different parameters for characterization of NPs and instruments used for these characterizations. ${ }^{23}$ Representative examples for different sources of the nanomaterials used for ciprofloxacin delivery and the characterization done in the articles included in this review are listed in Table 3. NP-based drug delivery systems introduce a wide range of therapeutics, by either binding the drug to their large surface area or carrying it to the site of infection effectively, safely, and with a controlled rate of targeted delivery. They are capable of disrupting bacterial membranes and hindering biofilm formation, thus reducing the survival of the microorganism. This way, NPs provide an alternative strategy with potential to use both antibiotic-free and antibiotic-coated approaches. ${ }^{10}$ Nanomaterial antibiotic functionalization includes use of polymers, dendrimers, and inorganic NPs with antibiotic molecules attached to their surfaces. This antibiotic nanomaterial functionalization results in improved effectiveness due to: 1) improved drug-

Table 2 Different Parameters for Characterization of Nanoparticles

\begin{tabular}{|l|l|}
\hline Nanoparticle Parameters & Instruments Used for Characterization \\
\hline Particle size and particle size distribution & Zeta sizer, laser diffractometry, PCS, mercury porosimetry \\
\hline Particle morphology & SEM, TEM, AFM \\
\hline Particle charge & Laser droplet anemometry, zeta potentiometer \\
\hline Surface hydrophobicity & $\begin{array}{l}\text { Measurement of water contact angle, X-ray photoelectron spectroscopy, hydrophobic interaction } \\
\text { chromatography }\end{array}$ \\
\hline Chemical analysis of surface & Static secondary ion mass spectrometry \\
\hline Carrier drug interaction & DSC \\
\hline Nanoparticle dispersion stability & Critical flocculation temperature \\
\hline Release profile & In-vitro release characteristic under physiologic and sink condition \\
\hline Drug stability & Bioassay \& chemoassay of drug extracted from nanoparticle \\
\hline
\end{tabular}

Notes: Reproduced from Patel S, Singh D, Srivastava S et al. Nanoparticles as a Platform for Antimicrobial Drug Delivery, Advances in Pharmacology and Pharmacy. 2017; 5(3): 31-43. Copyright $\bigcirc 2017$ by authors, all rights reserved. Authors agree that this article remains permanently open access under the terms of the Creative Commons Attribution License 4.0 International License. ${ }^{23}$.

Abbreviations: PCS, photon correlation spectroscopy; SEM, scanning electron microscopy; TEM, transmission electron microscopy; AFM, atomic force microscopy; DSC, differential scanning calorimetry. 
Table 3 Sources of Nanomaterials for Ciprofloxacin Delivery

\begin{tabular}{|l|l|l|c|}
\hline Nano-Ciprofloxacin Formulation & Source of Nanomaterial & Instruments for Characterization & Reference \\
\hline Chitosan-loaded ciprofloxacin & Purchased (synthetic) & DSC, DLS & [24] \\
\cline { 2 - 5 } & $\begin{array}{l}\text { Desert locusts, beetles, honey bee } \\
\text { exoskeletons, and shrimp shells }\end{array}$ & $\begin{array}{l}\text { Zeta sizer, FTIR, XRD, centrifugation, } \\
\text { UV-Vis spectroscopy }\end{array}$ & [25] \\
\hline Ciprofloxacin-loaded albumin & Human serum albumin & DLS, SEM, FTIR & [26] \\
\hline Ciprofloxacin in arginine nanocarriers & L-Arginine, protamine and pectin & Microscopy, FTIR, and zeta sizer \\
\hline Ciprofloxacin HCl-loaded CaCO ${ }_{3}$ NPs & Purchased & DLS, UV-Vis, zeta sizer, XRD, DSC, FTIR & [28] \\
\hline Ciprofloxacin incorporated PLGA & Purchased & Zeta sizer, TEM, SEM, HPLC, DSC & [29] \\
\hline Ciprofloxacin-loaded polysaccharide NPs & Purchased & $\begin{array}{l}\text { Zeta sizer, PCS, UV-Vis, FTIR, electron } \\
\text { microscopy }\end{array}$ & [30] \\
\hline Ciprofloxacin HCl-loaded SLNs & Purchased & $\begin{array}{l}\text { LLD, LDE, HPLC-UV, AUC, AFM, SEM, } \\
\text { TEM, }\end{array}$ \\
\hline Ciprofloxacin in liposomes & Lipids purchased & DLS, FES & [32] \\
\hline
\end{tabular}

Abbreviations: DSC, differential scanning calorimetry; FTIR, Fourier transform infrared; XRD; X-ray diffractometer; UV-Vis, ultraviolet-visible; DLS, dynamic light scattering; SEM, scanning electron microscopy; TEM, transmission electron microscopy; HPLC, high performance liquid chromatography; AUC, analytical ultra centrifuge; LLD, laser light diffraction; LDE, laser Doppler electrophoresis; AFM, atomic fluorescence microscopy; FES, fluorescence emission spectra; PCS, photon correlation spectroscopy.

delivery features, better than the conventional antibiotic alone; and 2) interesting synergistic effects. NMs themselves can damage the cell membrane, generate reactive oxygen species, and release toxic metals causing intrinsic antimicrobial effect. Rather than focusing upon particular biochemical processes as traditional antibiotics, they are likely to disrupt multiple cellular processes in a less specific fashion. ${ }^{9}$

\section{Nanotoxicity, Resistance, and Regulatory Aspects}

Besides their anticipated benefits, prolonged exposure to NMs might cause nanotoxicity and thus, possible jeopardies for human health and the ecosystems. Proteins and other biomolecules from the body and the environment could rapidly bind to NMs, resulting in the formation of protein/biomolecule corona that can affect NM's nature of interaction with the body system. Systematized knowledge on NMs toxicity is required for their rational design with reduced potential toxicity. ${ }^{33}$ NPs that are inhaled or extracted from drug-NP formulations can effortlessly reach the bloodstream and then the liver, heart, or blood cells. Many of them seem to be nontoxic and others have positive health effects. The engineered, smallest size of NPs can facilitate the translocation of active chemical species from barriers of the skin, lung, body tissues, and organs. Thus, irreversible oxidative stress, organelle damage, asthma, and cancer can be caused by NPs depending on their composition. The generation of neoantigens that cause possible organ enlargement and dysfunction are common chronic toxic effects of NPs. ${ }^{22}$

Even though there are not well established reports regarding the mechanism and intensity of nano-resistance to microorganisms, there are some predictions and pre-concerns. Potential novel antifungal metal-based NPs, such as $\mathrm{ZnO}$, $\mathrm{Ag}$, or $\mathrm{CuO}$, showed fungal resistance mediated by biomolecule coronas acquired in pathophysiological and ecological environments. ${ }^{34}$ The great diversity of antimicrobial mechanisms exerted by NPs suggests that bacteria are unlikely to generate resistance to NPs. However, bacterial resistance is dreaded with the mechanisms not yet been investigated more. There are predicted intrinsic and extrinsic mechanisms for NP microbial resistance. The intrinsic resistance mechanisms can include outer membrane permeability, multidrug resistance efflux pumps, downregulation of genes, and chromosomal resistance genes. In the other way, the extrinsic mechanisms are in the form of adaptive mutations, point mutations, and plasmids with resistance genes. It is important to study all the aforementioned resistance mechanisms to understand and prevent complex resistance challenges. ${ }^{35,36}$ 
In spite of the great hope on nanomedicine and its pharmaceutical applications, there is currently very little regulation that guides the area. However, the research community is calling repeatedly for providing legal certainty to the manufacturers, policymakers, healthcare providers, and the general public so as to minimize challenges in funding, research, and development of works on NMs that reduce public acceptance and perception. ${ }^{37}$ Regulations via legislation, laws, and rules have been implemented by several government organizations including the USA and the European Union (EU) to minimize or avoid risks associated with NMs. However, there is no specific international regulation, no internationally agreed upon protocols or legal definitions for production, handling or labeling, testing toxicity, and evaluating the environmental impact of NPs. Extensive research in the field of nanotoxicology and strict regulation by government agencies is essential to identify and avoid toxic NPs. ${ }^{22}$

\section{Nanotechnology-Based Approaches for Ciprofloxacin Delivery Nanoparticles of Chitosan-Loaded Ciprofloxacin}

Chitosan, the amino polysaccharide copolymer of 1,4-D-glucosamine and $N$-acetyl glucosamine is derived from chitin by alkaline or enzymatic deacetylation. It is used for encapsulation of many antibiotics for their nano-delivery. ${ }^{38}$ The minimum inhibitory concentration (MIC) of ciprofloxacin-loaded chitosan NPs was demonstrated to be $50 \%$ lower than that of ciprofloxacin $\mathrm{HCl}$ alone against $E$. coli and $S$. aureus. The loading resulted in increased drug penetration into the bacterial cell improving antibacterial activity with reduced dose. ${ }^{24}$ Another similar study indicated uppermost ciprofloxacin antibacterial activity against $E$. coli and MRSA with a significant (85.6\%) decrease in MIC, enhanced drug delivery, increased drug penetration, and controlled release. ${ }^{25}$ Ciprofloxacinchitosan microparticles for local drug delivery to the lungs against pneumonia causing agents, namely E. coli, P. aeruginosa, and S. aureus demonstrated good loading efficiency, enhanced localized effects, better escaping of the body's local defense system, improved antibacterial effects, and less cytotoxicity to human lung epithelial cells. ${ }^{39}$

In vitro evaluation of sustained ciprofloxacin release from chitosan/hydroxyapatite hydrogel nanocomposites exhibited a sustained antibacterial activity against $S$. aureus and E. coli through a diffusion process promising for designing prolonged release drug delivery systems. ${ }^{40}$ Other in vitro release data of ciprofloxacin with chitosan-alginate systems promised an alternative system for the sustained delivery of ciprofloxacin. ${ }^{19}$ A study on chitosan bandages loaded with ciprofloxacin for polymicrobial wound infections demonstrated good flexibility, tensile strength, porosity, and wound exudate absorption. The bandage released ciprofloxacin for up to 14 days in a sustained manner and showed a significant antimicrobial activity both in vitro and in vivo toward poly-microbial cultures of C. albicans, E. coli, and S. aureus. ${ }^{41}$ The other important investigation of chitosan-ciprofloxacin NPs is targeting. A study conducted on the ciprofloxacin capsule system encapsulated by chitosan and dextran sulfate biopolymers demonstrated improved targeting of the Salmonella containing vacuoles to kill the pathogen so that complete cure can be achieved without any relapse. ${ }^{42}$ In another study, ciprofloxacin was loaded into chitosan/heparin NPs to target enteropathogenic bacteria. The synergistic effect reduced the MIC of free ciprofloxacin against $E$. coli by half and a targeted killing of $60 \%$ load within $30 \mathrm{~min}^{43}$

\section{Ciprofloxacin-Loaded PLGA/PCL Nanoparticles}

The polymeric NPs of poly(D,L-lactide-coglycolide) (PLGA) and poly( $\varepsilon$-caprolactone) (PCL) have shown significant therapeutic potential to achieve controlled drug delivery. An investigation on the incorporation of ciprofloxacin encapsulated PLGA and PCL NPs on tissue-engineered scaffolds resulted in high encapsulation efficiency, better stability, predictable controlled release, and targeting the site of action. Additionally, both the encapsulated NPs were safer and effective against $S$. aureus and $P$. aeruginosa compared to the free drug. ${ }^{29}$ Ciprofloxacin-loaded PLGA electrospun fibers also demonstrated effective antibacterial properties against $P$. aeruginosa, $S$. aureus, and S. epidermidis. ${ }^{44}$ Türeli et al studied the encapsulation efficiency, release pattern, cytotoxicity, and antibacterial activity of ciprofloxacin-loaded PLGA NPs. The result revealed high drug loading, promising penetration into the mucus where the bacteria reside, controlled release, sustained local drug concentration, and enhanced anti- $P$. aeruginosa activity with reduced dose, thus reduced side effects. ${ }^{45} \mathrm{NP}$ parameter optimization enabled ciprofloxacin-loaded PLGA NP preparations with pre-defined quality to fulfill the requirements of local drug delivery under cystic fibrosis disease conditions. ${ }^{46}$ 
Similarly, a study on ciprofloxacin-loaded PLGA NPs for eradicating P. aeruginosa biofilm showed a steady release of the antibiotic and the repeated application of the NPs for 3 days resulted in biofilm reduction by $95 \% .{ }^{47}$ Evaluation of the efficacy of sustained release PLGA micro- and nanoparticles containing ciprofloxacin against bacterial biofilms demonstrated a sustained release over 6 days to effectively eradicate culturable $S$. aureus. The formulation might be a valuable alternative for the treatment of biofilms by achieving high local and sustained drug concentrations while minimizing systemic adverse effects and improving patient compliance. ${ }^{48}$

\section{Liposomal and Micellar NPs for Ciprofloxacin Delivery}

The efficacy of liposomal antibiotics has been shown in numerous in vivo studies to be clearly superior to that of the free antibiotics. But, formulation factors such as liposome's membrane rigidity, surface charge, size, and polymer hydrophobicity must be controlled and optimized. ${ }^{49}$ Liposomal ciprofloxacin formulations have been developed for enhancing lung residence time reducing the burden of inhaled antimicrobial therapy from multiple daily administrations due to rapid absorptive clearance of antibiotics from the lungs. ${ }^{50} \mathrm{In}$ a study comparing the release of ciprofloxacin from the liposomal formulation and a solution formulation for inhaled drug delivery, the liposomal formulation provided zero-order release kinetics that prolonged residence time in the lungs. ${ }^{51}$ Liposomal ciprofloxacin administered intravenously and intraperitoneally to mice infected with intracellular S. typhimurium demonstrated an increased plasma habitation time and concentration of drug in the liver, spleen, lungs, and kidneys. Besides, an intratracheal administration increased pulmonary drug retention. It has also an extended survival and can reduce the number of bacteria in the liver and spleen compared with the free drug. ${ }^{52}$ Mannosylated liposomes loaded with ciprofloxacin were investigated for targeting against intracellular infections of the respiratory system. Upon pulmonary administration on rats, they demonstrated an efficient macrophage targeting. ${ }^{53}$

Ciprofloxacin-loaded liposome against Salmonella Dublin demonstrated greater stability and antimicrobial properties than free ciprofloxacin. It helps the drug to be accumulated in the spleen and liver persisting for $48 \mathrm{~h}$ after the final administration. ${ }^{54}$ PEGylated phosphatidylcholine-rich liposomal nanovesicles loaded with ciprofloxacin for ameliorating infectious pneumonia led to the eradication of intracellular MRSA with a 3.2-fold increase of pulmonary ciprofloxacin accumulation after IV injection of the lipid nanovesicles. ${ }^{55}$ Inhaled liposomal ciprofloxacin NPs were developed to overcome the rapid clearance of antibiotics from the lungs. They successfully controlled the release of ciprofloxacin and showed enhanced antibacterial activity against $P$. aeruginosa supporting the potential of using inhaled liposomal ciprofloxacin for respiratory infections. ${ }^{56}$ Current Phase-II clinical studies demonstrated a dual release profile (a rapid bactericidal effect followed by a sustained release from liposomes). The clinical study reports indicated that they are well tolerated with no serious adverse effects, and they significantly reduced pseudomonal bacterial load. ${ }^{57}$

Enhanced localization of ciprofloxacin was achieved by actively loading it into photoactivatable liposomes that contain small amounts of porphyrin-phospholipid. High drug encapsulation and significant light-induced drug release was achieved which established the feasibility of remote loading antibiotics into photoactivatable liposomes promising for enhanced localized antibiotic therapy. ${ }^{32}$ Ciprofloxacin efficiently inhibits the growth of M. avium in vitro in a murine macrophage-like cell line using negatively charged liposomes and in vivo using specific stealth liposomes in a mouse model of tuberculosis infection. ${ }^{58}$ Ciprofloxacin-loaded liposomes coated with muco-adhesive biocompatible chitosan (chitosomes) resulted in a more pronounced effect against both Gram-positive and Gram-negative bacteria with a diffusional drug release mechanism. In addition, chitosan coating improved the ocular permeation of ciprofloxacin $\mathrm{HCl}$ where bacterial conjunctivitis symptoms were controlled by the prolonged release. ${ }^{59}$

Polymeric micelles entrap hydrophilic and hydrophobic compounds and charged macromolecules through electrostatic, hydrophobic, and hydrogen bonding interactions. ${ }^{60}$ The micellar ciprofloxacin treatment in P. aeruginosa infected mice showed on-demand release of ciprofloxacin with reduced bacterial load and alveolar injury. ${ }^{61}$ In an investigation to enhance ocular delivery of ciprofloxacin, micellar formulations had been used to compare them with commercially available ciprofloxacin eye drops in rabbit's eye. Pharmacokinetic parameters were determined and the investigated micellar formulations showed a significant improvement in the rate and extent of absorption with prolonged effect. ${ }^{62}$ Zadeh et al also confirmed that polymeric micelles with proper particle sizes below $190 \mathrm{~nm}$ demonstrated improved 


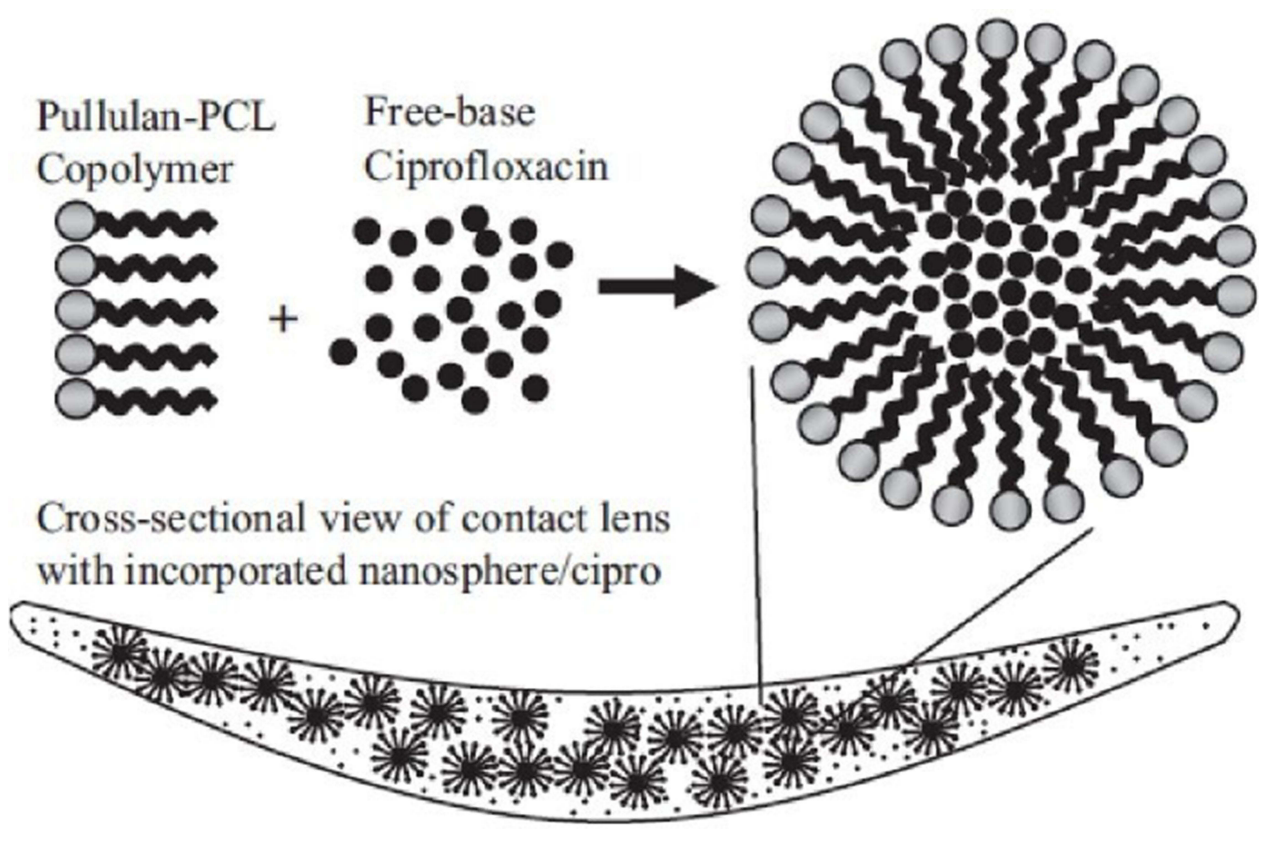

Figure I Micellar nanosphere encapsulated ciprofloxacin incorporated into contact lens

Notes: Reproduced with permission from Garhwal R, Shady SF, Ellis EJ, et al. Sustained ocular delivery of ciprofloxacin using nanospheres and conventional contact lens materials. Invest Ophthalmol Vis Sci. 2012;53:134I-1352. Copyright (C 2012 Association for Research in Vision and Ophthalmology. ${ }^{64}$

gastro-intestinal solubility, intestinal membrane permeability, and loading efficiency. ${ }^{63}$ A study on ciprofloxacin-loaded core-shell micellar nanospheres incorporated into a conventional, transparent contact lens provided a sustained, effective, and new drug delivery platform for treating ocular disorders. Nanosphere/ciprofloxacin incorporated into contact lenses (Figure 1) was tested for growth inhibition of $S$. aureus and P. aeruginosa and the result demonstrated that polymerized contact lenses were transparent and effective against the bacteria. ${ }^{64}$

\section{Other Polymer-Based Nanocarriers for Ciprofloxacin Delivery}

Albumin, as a polymer abundant in the human body, has favorable properties such as biodegradability, biocompatibility, nontoxicity, low cost, and high ability to bind to a variety of drugs. Ciprofloxacin-loaded human serum albumin (HSA) NPs showed a significant antibacterial effect with greater susceptibility to bacteria which was resistant to all antibiotic groups with sustained release, increased serum half-life, reduced drug toxicity and side effects, and increased susceptibility or reduced resistance. ${ }^{26}$ In another study, ciprofloxacin entrapped in an arginine-based nanocarrier system developed on a mesoporous silica NP (Arg-MSN) showed gradual drug release, two-fold higher in vitro antibacterial activity against Salmonella, targeting at the intra-vacuolar Salmonella, and localized delivery of the antibiotic. In addition, an increased reactive nitrogen species upon Arg-MSN treatment in the infected cells was observed. The coordinated effect of improved antibiotic delivery, intracellular targeting, and production of reactive nitrogen species resulted in an enhanced antibacterial activity (Figure 2). This can be an attractive antibiotic carrier system for clearing intravacuolar infections. ${ }^{27}$

The antibacterial effect of ciprofloxacin was also enhanced by encapsulating the drug in carboxymethyl tamarind kernel polysaccharide (CMTKP). Ciprofloxacin-loaded CMTKP NPs revealed higher antibacterial activity and lowest cytotoxicity than ciprofloxacin alone. ${ }^{30}$ Ciprofloxacin-loaded poly-isobutyl cyanoacrylate polymeric NPs demonstrated improved pharmacokinetic properties and augmented antibacterial activity against Mycobacterium avium. ${ }^{65}$ Bioactive materials like hydroxyapatite (HA) in combination with antibiotics are widely developed for the treatment of osteomyelitis which play a dual role: as bone substitutes at the affected site and as local drug delivery systems for antibiotic delivery. The in situ loading of ciprofloxacin onto HA NPs provided sustained and prolonged release of ciprofloxacin with synergistic antibacterial activity against $S$. aureus and $E$. coli that causes osteomyelitis which can suggest the synthesized ciprofloxacin-loaded HA 

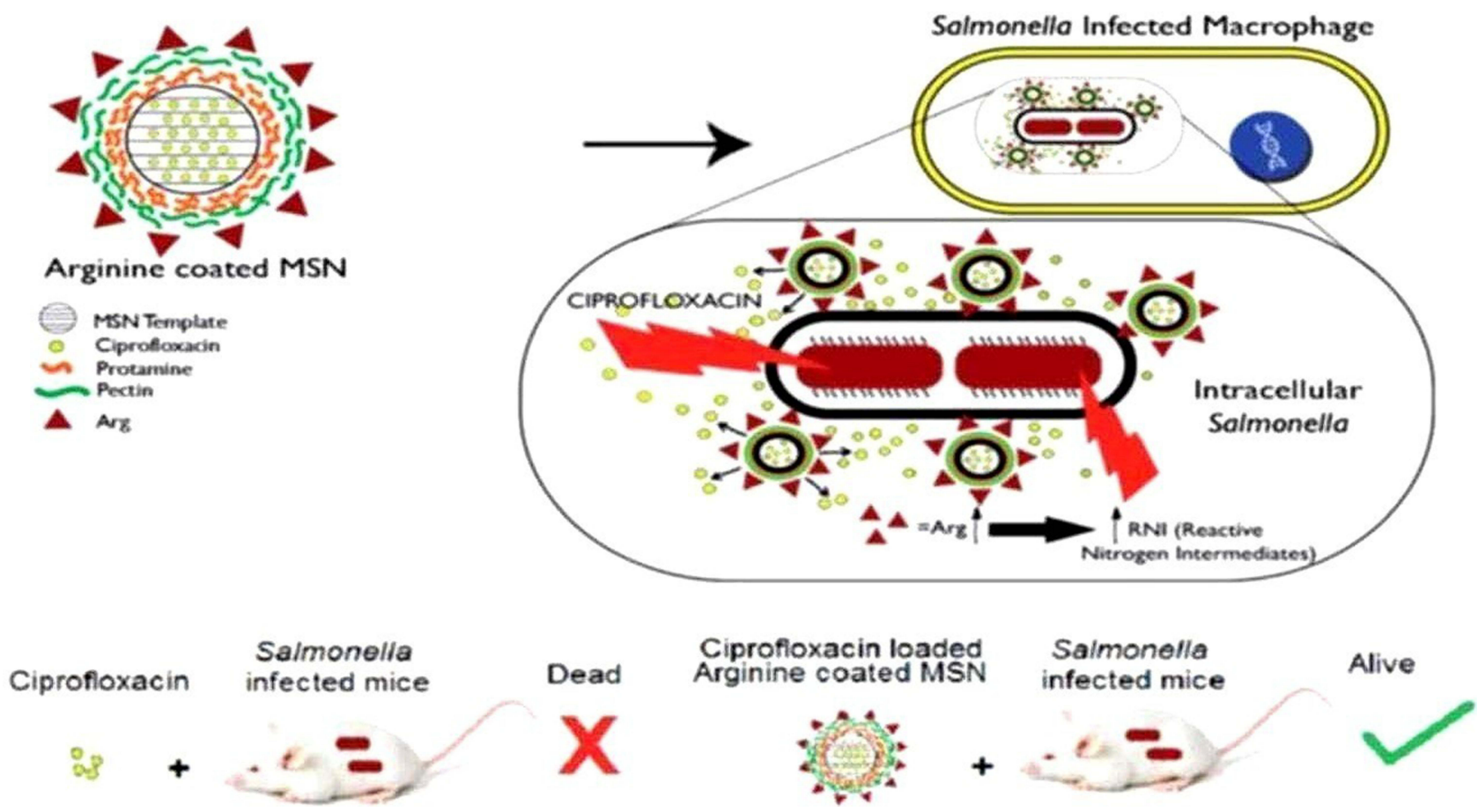

Figure 2 Arginine grafted mesoporous silica nanoparticle targeting intracellular Salmonella.

Notes: Reproduced from Mudakavi RJ, Vanamali S, Chakravortty D, and Raichur AM. Development of arginine based nanocarriers for targeting and treatment of intracellular Salmonella, RSC Adv, 2017;7:7022. This article is licensed under a Creative Commons Attribution 3.0 Unported Licence. $^{27}$

NPs as potential candidates for the treatment of osteomyelitis. ${ }^{66}$ Use of ciprofloxacin during tissue engineering practices can be enhanced by applying it as ciprofloxacin-loaded polymeric NP incorporated electrospun fiber. ${ }^{29}$

\section{Ciprofloxacin-Conjugated Metallic Nanoparticles}

NPs of silver $(\mathrm{Ag})$, gold $(\mathrm{Au})$, Zinc $(\mathrm{Zn})$, copper $(\mathrm{Cu})$, and inorganic carriers such as silica and alumina serve as novel carriers for pharmaceutical formulations. Their synergistic antibacterial activity with their bio-modification property with several functional groups such as amines, disulfides, and thiols is a considerable promising advantage. ${ }^{67}$ Metallic NPs can tackle bacterial threats passively through prolonged drug retention at the specific site, or actively through surface conjugation with active molecules that can bind to a certain target. ${ }^{10}$

Graphene oxide-coated Ag NPs demonstrated an effective encapsulation of ciprofloxacin with a controlled release and a synergistic antibiotic activity against selected bacterial strains of S. aureus, B. subtilis, E. coli, and P. aeruginosa. ${ }^{68}$ In an investigation of Ag-ciprofloxacin nanoformulation against Pseudomonas keratitis, a synergistic antibacterial effect, decreased ciprofloxacin dose, and decreased genetic mutations for decreasing in the emergence of resistant strains were the main findings. ${ }^{69}$ Functionalization with ciprofloxacin after a one year aging period of synthesized Ag NPs facilitated the penetration of $\mathrm{Ag}$ ions into the bacterial cell wall which resulted in a significant improvement of antibacterial activity against both Gram-positive and Gram-negative bacteria. ${ }^{70}$ In another study, ciprofloxacin conjugated to biogenic Au NPs demonstrated enhanced bactericidal activity against Gram-negative and Gram-positive bacteria. ${ }^{71}$ Ciprofloxacin, adsorbed in Au NPs, showed a characteristic release over an extended period of time. ${ }^{72}$ Ciprofloxacin capping with metallic ( $\mathrm{Ag}$ and $\mathrm{Au}$ ) NPs under optimized conditions significantly implicated an improved solubility, pharmacokinetics, bioavailability, and a significant antibacterial activity. ${ }^{73}$

Banoee et al investigated that the antibacterial activity of ciprofloxacin against $S$. aureus and E. coli increased in the presence of ZnO NPs. The study predicted that ZnO NPs may interfere with the efflux pumping activity which helps for restoring ciprofloxacin action by increasing its absorption into the cell. ${ }^{74}$ In another similar study, only the low concentrations of ciprofloxacin-coated ZnO NPs (equivalent to the sub-minimum inhibitory concentrations of pure ciprofloxacin) demonstrated a considerably enhanced (4- to 32-fold) antibacterial activity. This result is of particular 


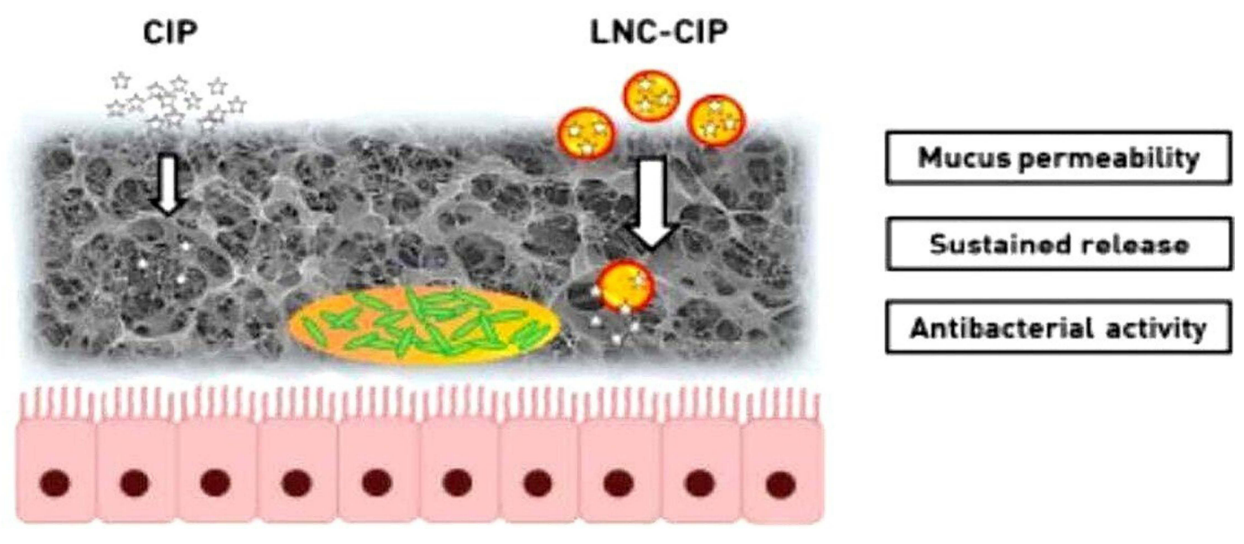

Figure 3 Ciprofloxacin-loaded lipid-core nanocapsules and their effects. ${ }^{31}$

Notes: Reproduced from Torge A, Wagner S, Chaves PS, et al. Ciprofloxacin-loaded lipid-core nanocapsules as mucus penetrating drug delivery system intended for the treatment of bacterial infections in cystic fibrosis. Int J Pharm. 2017;527(I-2):92-102. Copyright 2017, with permission from Elsevier.

value, as using biocompatible $\mathrm{ZnO}$ NPs in combination therapy can lower amounts of antibiotics needed. ${ }^{75}$ A review report by Allahverdiyev et al stated that ciprofloxacin with ZnO NPs showed increased inhibition zone areas against $S$. aureus and E. coli. The effect of $\mathrm{ZnO}$ is proposed to be interference with the pumping activity of NorA protein, possiblly binding on the nitogen, florine, and carboxilic ends of the ciprofloxacin structure (Figure 3) ${ }^{76}$

Metal oxide NPs are already applied as an antibacterial additive in various products. Nanostructured $\mathrm{MgO}$ is a low cost, easy to manipulate, and biocompatible metal oxide with intrinsic antibacterial activity. Mixed biphasic $\mathrm{ZnMgO} \mathrm{NPs}$ showed a high antibacterial efficiency. $\mathrm{Zn}_{0.15} \mathrm{Mg}_{0.85} \mathrm{O}$ combined with ciprofloxacin potentiated antibiotic activity of ciprofloxacin against $B$. subtilis and E. coli. ${ }^{77}$ Metal oxides with magnetic drug delivery properties, like iron oxide, improve the ability to define specific locations increasing the target and reducing the nontarget drug concentration. Ciprofloxacin-loaded $\mathrm{CoFe}_{2} \mathrm{O}_{4}$ led to magnetic targeting of bacteria, a controlled manner drug release, and a 2-fold increase in the inhibition zone. ${ }^{68,78}$ A study of ciprofloxacin-loaded $\mathrm{CaCO}_{3} \mathrm{NPs}$ showed slower in vitro release of 12 $\mathrm{h}$ and a preserved antimicrobial effect against $S$. aureus after 2 days of incubation. ${ }^{28}$

\section{Ciprofloxacin-Loaded Solid Lipid Nanocarriers}

Encapsulation and drug delivery with lipid nanocarriers prevents antibiotic drug therapies suffering from issues of over dosage, associated side effects, poor patient compliance, and development of resistances. ${ }^{20}$ While poor membrane transport limits the potency of many antibiotics, drug-loaded NP vehicles can enter host cells via endocytosis. Besides, the carrying potentials of multiple drug combinations offer highly complex and unpredictable antimicrobial actions where the bacteria cannot develop resistance. ${ }^{10,79}$ The possible improvement in the antimicrobial therapy of ciprofloxacin-loaded lipid NPs is due to the enhanced intracellular delivery of the poorly-cell-penetrating antibiotics. The NPs are ingested by the phagocytic system, involve macrophage activation, and increase the immune response of the host. ${ }^{80}$ Solid lipid nanoparticles (SLNPs) represent a superior alternative to other nanocarriers due to their high entrapment efficiency, ease of scale up, stability during storage, protection of labile drugs against degradation, improved tissue tolerance, less stringent regulatory requirements owing to the use of physiological lipids, and delivery by almost all routes. ${ }^{81,82}$

Ciprofloxacin-loaded SLNP formulations were subjected to an in vitro microbiological assay to verify the effect of nanoencapsulation on the cell growth inhibitory activity against $S$. aureus and $P$. aeruginosa. The result showed a more efficacious delivery inside bacterial cells and a higher stability of the encapsulated drug. ${ }^{83}$ SLNPs are effective against skin infections as they tend to adhere to the skin surface and continuously release drugs over long periods of time in the stratum corneum. Ciprofloxacin-loaded SLNPs exhibit the prolonged release of drugs, especially in the skin and oral infections. A comparative study of ciprofloxacin-loaded protein, lipid, and chitosan NPs showed that SLNs were found to produce controlled release with the smallest size than others with the potential to act as long-acting antibiotic carriers in 
various regional and systemic infectious states. ${ }^{84}$ Ghaffari et al demonstrated that ciprofloxacin release from SLNPs is more rapid with a significant burst effect followed by a sustained release that suggested that SLNPs can act as promising carriers for sustained ciprofloxacin release. ${ }^{19}$

Ciprofloxacin release from SLNPs containing stearic acid displayed strongest burst effect with rapid release rate. ${ }^{85}$ In an investigation for a solution to overcome biological (mucus) barrier, ciprofloxacin was loaded into lipid-core nanocapsules (LNC) for high mucus permeability, sustained release, and antibacterial activity. It resulted in $50 \%$ increase on drug permeation through mucus. High encapsulation efficiency, improved apparent aqueous solubility, reduced dosing frequency and overall dose, biofilm preventing, improved mucus permeation, and protection of the drug from deactivation were the major observations of the study. With these combined advantages, ciprofloxacinloaded LNC represented a promising drug delivery system of an improved antibiotic therapy in cystic fibrosis. ${ }^{31}$

\section{Conclusion}

Targeting ciprofloxacin more precisely to the site of infection by using carriers, an adjuvant approach whereby ciprofloxacin is paired with compounds that potentiate its activity against biofilm cells, combination of materials that act synergistically with ciprofloxacin against microbes, and protection of the drug from deactivation or efflux have been demonstrated to combat resistance and enhance antibacterial activity. Ciprofloxacin-NP conjugation, ciprofloxacin nanoencapsulation, loading ciprofloxacin in liposomes, and polymeric nanoformations are among the different promising nanotechnology-based approaches to combat ciprofloxacin resistance. Highly reduced MIC and dosing, reduced side effects, enhanced stability, targeting and controlling release, synergistic effect, optimized pharmacokinetic profile, and minimized chance of resistance have been demonstrated from the different ciprofloxacin nano-delivery studies. Especially, the synergistic activity, biodegradability, biocompatibility, higher stability, simple synthesis pathway, high loading efficiency, and cost-effectiveness of polymeric NPs are very promising and interesting for further investigations and alternative-setting approaches.

\section{Abbreviations}

AMR, antimicrobial resistance; CMTKP, carboxymethyl tamarind kernel polysaccharide; HA, hydroxyapatite; LNC, lipid-core nanocapsules; MDR, multidrug resistant; MIC, minimum inhibitory concentration; MRSA, methicillinresistant S. aureus; MSSA, methicillin-susceptible S. aureus; NMs, nanomaterials; NPs, nanoparticles; PCL, poly $(\varepsilon-$ caprolactone); PLGA, poly(lactic-co-glycolic) acid; SLN, solid lipid nanocarrier; SLNPs, solid lipid nanoparticles; UTI, urinary tract infections.

\section{Acknowledgments}

Addis Ababa University is thankfully acknowledged for giving us an internet access.

\section{Funding}

No funding available.

\section{Disclosure}

The authors have declared that there is no competing interest.

\section{References}

1. World Heath Organization. Bibliography of scientific publications on antimicrobial resistance from South-East Asia Region, Printed in India. (C) World Health Organization; 2011.

2. Gashe F, Mulisa E, Mekonnen M, Zeleke G. Antimicrobial resistance profile of different clinical isolates against third-generation cephalosporins. J Pharmaceut. 2018;2018:1-7. doi:10.1155/2018/5070742

3. Pham TDM, Ziora ZM, Blaskovich MAT. Quinolone Antibiotics. Med Chem Commun. 2019;10(10):1719-1739. doi:10.1039/C9MD00120D

4. Sharma D, Patel RP, Zaidi STR, Sarker MMR, Lean QY, Ming LC. Interplay of the quality of ciprofloxacin and antibiotic resistance in developing countries. Front Pharmacol. 2017;8:546. doi:10.3389/fphar.2017.00546

5. Vidyavathi M, Srividya G. A review on ciprofloxacin: dosage form perspective. Int J Appl Pharmaceut. 2018;10(4):6. doi:10.22159/ ijap.2018v10i4.25315 
6. Reis ACC, Santos SRS, Souza SC, Saldanha MG, Pitanga TN, Oliveira RR. Ciprofloxacin resistance pattern among bacteria isolated from patients with community-acquired urinary tract infection. Rev Inst Med Trop Sao Paulo. 2016;58:53. doi:10.1590/S1678-9946201658053

7. Mulder M, de Jong JCK, Goessens WHF, et al. Risk factors for resistance to ciprofloxacin in community-acquired urinary tract infections due to Escherichia coli in an elderly population. J Antimicrob Chemother. 2017;72:281-289. doi:10.1093/jac/dkw399

8. Lee C-R, Cho I, Jeong B, Lee S. Strategies to minimize antibiotic resistance. Int J Environ Res Public Health. 2013;10(9):4274-4305.

9. Aruguete DM, Kim B, Hochella MF, et al. Antimicrobial nanotechnology: its potential for the effective management of microbial drug resistance and implications for research needs in microbial nanotoxicology. Environ Sci Processes Impacts. 2013;15(1):93-102.

10. Baptista PV, McCusker MP, Carvalho A, et al. Nano-strategies to fight multidrug resistant bacteria - "A Battle of the Titans". Front Microbiol. 2018;9:1441.

11. Rehman A, Patrick WM, Lamont IL. Mechanisms of ciprofloxacin resistance in Pseudomonas aeruginosa: new approaches to an old problem. J Med Microbiol. 2019;68(1):1-10. doi:10.1099/jmm.0.000873

12. Scheld WM. Maintaining fluoroquinolone class efficacy: review of influencing factors. Emerg Infect Dis. 2003;9(1). doi:10.3201/eid0901.020277

13. Ahmad I, Ahmad S, Rumbaugh KP, Editors. Antibacterial Drug Discovery to Combat MDR. Singapore: (C) Springer Nature Singapore Pte Ltd; 2019.

14. Hamed SM, Elkhatib WF, El-Mahallawy HA, Helmy MM, Ashour MS, Aboshanab KMA. Multiple mechanisms contributing to ciprofloxacin resistance among gram negative bacteria causing infections to cancer patients. Sci Rep. 2018;8(1):12268. doi:10.1038/s41598-018-30756-4

15. Campion JJ, McNamara JJ, Evans ME. Evolution of ciprofloxacin-resistant Staphylococcus aureus in in vitro pharmacokinetic environments. Antimicrob Agents Chemother. 2004;48(12):4733-4744. doi:10.1128/AAC.48.12.4733-4744.2004

16. Cirz RT, Romesberg FE. Induction and inhibition of ciprofloxacin resistance-conferring mutations in hypermutator bacteria. Antimicrob Agents Chemother. 2006;50(1):220-225. doi:10.1128/AAC.50.1.220-225.2006

17. Zhao F, Yang H, Bi D, Khaledi A, Qiao M. A systematic review and meta-analysis of antibiotic resistance patterns, and the correlation between biofilm formation with virulence factors in uropathogenic E. coli isolated from urinary tract infections. Microb Pathog. 2020;144:104196. doi:10.1016/j.micpath.2020.104196

18. O'Regan E, Quinn T, Frye JG, et al. Fitness costs and stability of a high-level ciprofloxacin resistance phenotype in Salmonella enterica serotype Enteritidis: reduced infectivity associated with decreased expression of Salmonella pathogenicity Island 1 genes. Antimicrob Agents Chemother. 2010;54(1):367-374. doi:10.1128/AAC.00801-09

19. Ghaffari S, Varshosaz J, Haririan I, Khoshayand MR, Azarmi S, Gazori T. Ciprofloxacin loaded Alginate/Chitosan and solid lipid nanoparticles, preparation, and characterization. J Dispers Sci Technol. 2012;33(5):685-689. doi:10.1080/01932691.2011.579831

20. Kumar S, Bhanjana G, Kumar A, Taneja K, Dilbaghi N, Kim K-H. Synthesis and optimization of ceftriaxone-loaded solid lipid nanocarriers. Chem Phys Lipids. 2016;200:126-132. doi:10.1016/j.chemphyslip.2016.09.002

21. Bali GK, Singla S, Kashyap Y, et al. Preparation, physico-chemical characterization and pharmacodynamics of ceftriaxone loaded BSA nanoparticles. J Nanomed Nanotechnol. 2018;9:509. doi:10.4172/2157-7439.1000509

22. Jaison J, Barhoum A, Chan YS, Dufresne A, Danquah MK. Review on nanoparticles and nanostructured materials: history, sources, toxicity and regulations. Beilstein J Nanotechnol. 2018;9:1050-1074. doi:10.3762/bjnano.9.98

23. Patel S, Singh D, Srivastava S, et al. Nanoparticles as a platform for antimicrobial drug delivery. Adv Pharmacol Pharm. 2017;5(3):31-43. doi:10.13189/app.2017.050301

24. Sobhani Z, Samani SM, Montaseri H, Khezri E. Nanoparticles of chitosan loaded ciprofloxacin: fabrication and antimicrobial activity. Adv Pharm Bull. 2017;7(3):427-432. doi:10.15171/apb.2017.051

25. Marei N, Elwahy AHM, Salah TA, El Sherif Y, El-Samie EA. Enhanced antibacterial activity of Egyptian local insects' chitosan-based nanoparticles loaded with ciprofloxacin-HCl. Biomac. 2019;126:262-272.

26. Shafiei S, Hassanshahian M, Shakeri S, Hamayeli H. Evaluation the antibacterial activity of nanoantibiotics imipenem and ciprofloxacin loaded in human serum albumin against some antibiotic-resistant pathogenic bacteria. J Exper Nanosci. 2020;15(1):350-362. doi:10.1080/ 17458080.2020 .1796978

27. Mudakavi RJ, Vanamali S, Chakravortty D, Raichur AM. Development of arginine based nanocarriers for targeting and treatment of intracellular Salmonella. RSC Adv. 2017;7(12):7022. doi:10.1039/C6RA27868J

28. Dizaj SM, Lotfipour F, Barzegar-Jalali M, Zarrintan M, Adibkia K. Ciprofloxacin HCl-loaded calcium carbonate nanoparticles: preparation, solid state characterization, and evaluation of antimicrobial effect against. Artif Cells Nanomed Biotechnol. 2017;45(3):535-543. doi:10.3109/ 21691401.2016 .1161637

29. Günday C, Anand S, Gencer HB, et al. Ciprofloxacin-loaded polymeric nanoparticles incorporated electrospun fibers for drug delivery in tissue engineering applications. Drug Deliv Transl Res. 2020;10(3):706-720. doi:10.1007/s13346-020-00736-1

30. Dilbaghi N, Kaur H, Ahuja M, Arora P, Kumar S. Synthesis and evaluation of ciprofloxacin-loaded carboxymethyl tamarind kernel polysaccharide nanoparticles. $J$ Exper Nanosci. 2014;9(10):1015-1025. doi:10.1080/17458080.2013.771244

31. Torge A, Wagner S, Chaves PS, et al. Ciprofloxacin-loaded lipid-core nanocapsules as mucus penetrating drug delivery system intended for the treatment of bacterial infections in cystic fibrosis. Int J Pharm. 2017;527(1-2):92-102. doi:10.1016/j.ijpharm.2017.05.013

32. Ghosh S, Qi R, Carter KA, Zhang G, Pfeifer BA, Lovell JF. Loading and releasing ciprofloxacin in photoactivatable liposomes. Biochem Eng J. 2019;141:43-48. doi:10.1016/j.bej.2018.10.008

33. Westmeier D, Siemer S, Vallet C, et al. Boosting nanotoxicity to combat multidrug-resistant bacteria in pathophysiological environments. Nanoscale Adv. 2020;2(11):5428-5440. doi:10.1039/D0NA00644K

34. Siemer S, Westmeier D, Vallet C, et al. Resistance to nano-based antifungals is mediated by biomolecule coronas. ACS Appl Mater Interfaces. 2019;11(1):104-114. doi:10.1021/acsami.8b12175

35. Niño-Martínez N, Orozco NFS, Martínez-Castañón G-A, Méndez FT, Ruiz F. Molecular mechanisms of bacterial resistance to metal and metal oxide nanoparticles. Int J Mol Sci. 2019;20(11):2808. doi:10.3390/ijms20112808

36. Salas-Orozco M, Niño-Martínez N, Martínez-Castañón G-A, et al. Mechanisms of resistance to silver nanoparticles in endodontic bacteria: a literature review. J Nanomater. 2019;2019. doi:10.1155/2019/7630316

37. Foulkes R, Man E, Thind J, Yeung S, Joy A, Hoskins C. The regulation of nanomaterials and nanomedicines for clinical application: current and future perspectives. Biomater Sci. 2020;8(17):4653-4664. doi:10.1039/D0BM00558D 
38. Manimekalai P, Manavalan R. Selection of excipients for the formulation of Ceftriaxone sodium loaded chitosan nanoparticle through drug-excipient compatibility testing. Int $J$ PharmTech Res. 2015;8(1):5-10.

39. Kucukoglu V, Uzuner H, Kenar H, Karadenizli A. In vitro antibacterial activity of ciprofloxacin loaded chitosan microparticles and their effects on human lung epithelial cells. Int J Pharm. 2019;569:118578. doi:10.1016/j.ijpharm.2019.118578

40. Mahdavinia GR, Karimi MH, Soltaniniya M, Massoumi B. In vitro evaluation of sustained ciprofloxacin release from $\kappa$-carrageenan-crosslinked chitosan/hydroxyapatite hydrogel nanocomposites. Int J Biol Macromol. 2019;126:443-453. doi:10.1016/j.ijbiomac.2018.12.240

41. Raveendran NT, Mohandas A, Menon RR, Menon AS, Biswas R, Jayakumar R. Ciprofloxacin and fluconazole containing fibrin nanoparticles incorporated chitosan bandages for the treatment of polymicrobial wound infections. ACS Appl Bio Mater. 2018;2(1):243-254.

42. Gnanadhas DP, Thomas MB, Elango M, Raichur AM, Chakravortty D. Chitosan-dextran sulphate nanocapsule drug delivery system as an effective therapeutic against intraphagosomal pathogen Salmonella. J Antimicrob Chemother. 2013;68(11):2576-2586. doi:10.1093/jac/dkt252

43. Kumar GV, Su CH, Velusamy P. Ciprofloxacin loaded genipin cross-linked chitosan/heparin nanoparticles for drug delivery application. Mater Lett. 2016;180:119-122. doi:10.1016/j.matlet.2016.05.108

44. Buck E, Maisuria V, Tufenkji N, Cerruti M. Antibacterial properties of PLGA electrospun scaffolds containing ciprofloxacin incorporated by blending or physisorption. ACS Appl Bio Mater. 2018;1(3):627-635. doi:10.1021/acsabm.8b00112

45. Türeli NG, Torge A, Juntke J, et al. Ciprofloxacin-loaded PLGA nanoparticles against cystic fibrosis $P$. aeruginosa lung infections. Eur J Pharmaceut Biopharmaceut. 2017;17:363-371.

46. Türeli NG, Türeli AE, Schneider M. Optimization of ciprofloxacin complex loaded PLGA nanoparticles for pulmonary treatment of cystic fibrosis infections: design of experiments approach. Int J Pharm. 2016;515(1-2):343-351.

47. Baelo A, Levato R, Julián E, Crespo A, Astola J, Gavaldà J. Disassembling bacterial extracellular matrix with DNase-coated nanoparticles to enhance antibiotic delivery in biofilm infections. J Control Release. 2015;209:150-158. doi:10.1016/j.jconrel.2015.04.028

48. Thomas N, Thorn C, Richter K, Thierry B, Prestidge C. Efficacy of poly-lactic-co-glycolic acid micro- and nanoparticles of ciprofloxacin against bacterial biofilms. J Pharm Sci. 2016;105:1-8.

49. Hadinoto K, Cheow WS. Nano-antibiotics in chronic lung infection therapy against Pseudomonas aeruginosa. Colloids Surf B Biointerfaces. 2014;116:772-785. doi:10.1016/j.colsurfb.2014.02.032

50. Cipolla D, Wu H, Eastman S, Redelmeier R, Gonda I, Chan H. Development and characterization of an in vitro release assay for liposomal ciprofloxacin for inhalation. J Pharm Sci. 2014;103(1):314-327. doi:10.1002/jps.23795

51. Ong HX, Benaouda F, Traini D, et al. In vitro and ex vivo methods predict the enhanced lung residence time of liposomal ciprofloxacin formulations for nebulization. Eur J Pharmaceut Biopharmaceut. 2014;86(1):83-89. doi:10.1016/j.ejpb.2013.06.024

52. Page-Clisson ME, Pinto-Alphandary H, Chachaty E, Couvreur P, Andremont A. Drug targeting by polyalkylcyanoacrylate nanoparticles is not efficient against persistent Salmonella. Pharm Res. 1998;15(4):544-549. doi:10.1023/A:1011921608964

53. Chono S, Tanino T, Seki T, Morimoto K. Efficient drug targeting to rat alveolar macrophages by pulmonary administration of ciprofloxacin incorporated into mannosylated liposomes for treatment of respiratory intracellular parasitic infections. J Control Release. 2008;127(1):50-58. doi:10.1016/j.jconrel.2007.12.011

54. Magallanes M, Dijkstra J, Fierer J. Liposome-incorporated ciprofloxacin in treatment of murine salmonellosis. Antimicrob Agents Chemother. 1993;37(11):2293-2297. doi:10.1128/AAC.37.11.2293

55. Hsu CY, Sung CT, Aljuffali IA, Chen CH, Hu KY, Fang JY. Intravenous anti-MRSA phosphatiosomes mediate enhanced affinity to pulmonary surfactants for effective treatment of infectious pneumonia. Nanomed Nanotechnol Biol Med. 2018;14(2):215-225. doi:10.1016/j. nano.2017.10.006

56. Ong HX, Traini D, Cipolla D, et al. Liposomal nanoparticles control the uptake of ciprofloxacin across respiratory epithelia. Pharm Res. 2012;29:3335-3346.

57. Antoniu SA. Inhaled ciprofloxacin for chronic airways infections caused by Pseudomonas aeruginosa. Expert Rev Anti Infect Ther. 2012;10 (12):1439-1446. doi:10.1586/eri.12.136

58. Yanagihara K. Design of anti-bacterial drug and anti-mycobacterial drug for drug delivery system. Curr Pharm Des. 2002;8(6):475-482. doi: $10.2174 / 1381612023395808$

59. Mehanna MM, Elmaradny HA, Samaha MW. Mucoadhesive liposomes as ocular delivery system: physical, microbiological, and in vivo assessment Mucoadhesive liposomes in ocular delivery. Drug Dev Ind Pharm. 2010;36(1):108-118. doi:10.3109/03639040903099751

60. Zhang J, Ma PX. Host-guest interaction mediated polymeric core-shell assemblies: versatile nanocarriers for drug delivery. Angew Chem Int Ed. 2009;48(5):964-968. doi:10.1002/anie.200804135

61. Chen M, Xie S, Wei J, Song X, Ding Z, Li X. Antibacterial micelles with vancomycin-mediated targeting and $\mathrm{pH} /$ lipase-triggered release of antibiotics. ACS Appl Mater Interfaces. 2018;10(43):36814-36823. doi:10.1021/acsami.8b16092

62. Taha EI, Badran MM, El-Anazi MH, Bayomi MA, El-Bagory IM. Role of pluronic F127 micelles in enhancing ocular delivery of ciprofloxacin. J Mol Liq. 2014;199(2014):251-256. doi:10.1016/j.molliq.2014.09.021

63. Zadeh M, Sharif B, Esfahani G, Salimi Y. Permeability of ciprofloxacin-loaded polymeric micelles including ginsenoside as P-glycoprotein inhibitor through a Caco-2 cells monolayer as an intestinal absorption model. Molecules. 2018;23(8):1904. doi:10.3390/molecules23081904

64. Garhwal R, Shady SF, Ellis EJ, et al. Sustained ocular delivery of ciprofloxacin using nanospheres and conventional contact lens materials. IOVS. 2012;53(3):1341-1352.

65. Fawaz F, Bonini F, Maugein J, Lagueny AM. Ciprofloxacin-loaded polyisobutylcyanoacrylate nanoparticles: pharmacokinetics and in vitro antimicrobial activity. Int J Pharm. 1998;168(2):255-259. doi:10.1016/S0378-5173(98)00116-1

66. Kumar GS, Govindan R, Girija EK. In situ synthesis, characterization and in vitro studies of ciprofloxacin loaded hydroxyapatite nanoparticles for the treatment of osteomyelitis. J Mater Chem B. 2014;2(31):5052-5060. doi:10.1039/c4tb00339j

67. Panda BP, Patnaik S, Maharana RL. Current trends in design and development of nanopharmaceutical dosage forms. Bioequiv Bioavailab Int J. 2017;1(1):000104.

68. Kooti M, Sedeh AN, Motamedi H, Rezatofighi SE. Magnetic graphene oxide inlaid with silver nanoparticles as antibacterial and drug delivery composite. Appl Microbiol Biotechnol. 2018;102(8):3607-3621. doi:10.1007/s00253-018-8880-1

69. Zavarshani M, Ahmadi M, Saei HD, Tehrani AA, Naghadeh BD. Comparison therapeutic effects of ciprofloxacin, silver nanoparticles and their combination in the treatment of Pseudomonas keratitis in rabbit: an experimental study. Iran J Pharmaceut Res. 2019;18(1):320-327. 
70. Mohsen E, El-Borady OM, Mohamed MB, Fahim IS. Synthesis and characterization of ciprofloxacin loaded silver nanoparticles and investigation of their antibacterial effect. J Radiat Res Appl Sci. 2020;13(1):416-425.

71. Sreedharan SM, Singh R. Ciprofloxacin functionalized biogenic gold nanoflowers as nanoantibiotics against pathogenic bacterial strains. Int J Nanomedicine. 2019;14:9905-9916.

72. Tom RT, Suryanarayanan V, Reddy PG, Baskaran S, Pradeep T. Ciprofloxacin-protected gold nanoparticles. Langmuir. 2004;20:1909-1914.

73. Nisar M, Khan SA, Qayum M, et al. Robust synthesis of ciprofloxacin-capped metallic nanoparticles and their urease inhibitory assay. Molecules. 2016;21:411.

74. Banoee M, Seif S, Nazari ZE, et al. ZnO nanoparticles enhanced antibacterial activity of ciprofloxacin against Staphylococcus aureus and Escherichia coli. J Biomed Mater Res B Appl Biomater. 2010;93:557.

75. Seif S, Kazempour ZB, Pourmand MR, et al. Preparation of ciprofloxacin-coated zinc oxide nanoparticles and their antibacterial effects against clinical isolates of Staphylococcus aureus and Escherichia coli. Arzneimittelforschung. 2011;61(8):472-476.

76. Allahverdiyev AM, Kon KV, Abamor ES, Bagirova M, Rafailovich M. Coping with antibiotic resistance: combining nanoparticles with antibiotics and other antimicrobial agents. Expert Rev Anti Infect Ther. 2012;9(11):1035-1052.

77. Auger S, Henry C, Péchoux C, et al. Exploring multiple effects of $\mathrm{Zn}_{0.15} \mathrm{Mg}_{0.85} \mathrm{O}$ nanoparticles on Bacillus subtilis and macrophages. Sci Rep. 2018;8:12276.

78. Rodrigues GR, López-Abarrategui C, de la Serna Gómez I, Dias SC, Otero-González AJ, Franco OL. Antimicrobial magnetic nanoparticles based-therapies for controlling infectious diseases. Int J Pharm. 2018;555:356-367.

79. El-Salamouni NS, Farid RM, El-Kamel AH, El-Gamal SS. Effect of sterilization on the physical stability of brimonidine-loaded solid lipid nanoparticles and nanostructured lipid carriers. Int J Pharm. 2015;496(2):976-983.

80. Zaki NM, Hafez MM. Enhanced antibacterial effect of ceftriaxone sodium-loaded chitosan nanoparticles against intracellular Salmonella typhimurium. AAPS PharmSciTech. 2012;13(2):411-421.

81. Mehnert W. Solid lipid nanoparticles production, characterization and applications. Adv Drug Deliv Rev. 2001;47(2-3):165-196.

82. Qu J, Zhang L, Chen Z, et al. Nanostructured lipid carriers, solid lipid nanoparticles, and polymeric nanoparticles: which kind of drug delivery system is better for glioblastoma chemotherapy? Drug Deliver. 2016;23(9):3408-3416.

83. Pignatello R, Leonardi A, Fuochi V, Petronio G, Greco AS, Furneri PM. A method for efficient loading of ciprofloxacin hydrochloride in cationic solid lipid nanoparticles: formulation and microbiological evaluation. Nanomater. 2018;8:304.

84. Jain D, Banerjee R. Comparison of ciprofloxacin HCl-loaded protein, lipid, and chitosan nanoparticles for drug delivery. $J$ Biomed Mater Res B Appl Biomater. 2008;86B(1):105-112.

85. Shazly GA. Ciprofloxacin controlled-solid lipid nanoparticles: characterization, in vitro release, and antibacterial activity assessment. Biomed Res Int. 2017;2017:2120734.

\section{Publish your work in this journal}

Infection and Drug Resistance is an international, peer-reviewed open-access journal that focuses on the optimal treatment of infection (bacterial, fungal and viral) and the development and institution of preventive strategies to minimize the development and spread of resistance. The journal is specifically concerned with the epidemiology of antibiotic resistance and the mechanisms of resistance development and diffusion in both hospitals and the community. The manuscript management system is completely online and includes a very quick and fair peer-review system, which is all easy to use. Visit http://www.dovepress.com/testimonials.php to read real quotes from published authors.

Submit your manuscript here: https://www.dovepress.com/infection-and-drug-resistance-journal 